\title{
AUTHOR INDEX Volume 15
}

Abbassi, A.H., see Razmi

Abdullin, S., see Dželalija

Abe, H., Miguchi, H. \& Muta,

T., Dynamical fermion masses under the influence of Kaluza-Klein fermions in extra dimensions

Abud, M., Buccella, F.,

Falcone, D., Ricciardi, G. \& Tramontano, F., Neutrino masses and mixings in $\mathrm{SO}(10)$

Adam, C., Muratori, B. \& Nash, C., Zero modes in finite range magnetic fields

Agaev, S.S., Mukhtarov, A.I. \& Mamedova, Y.V., Mesons distribution amplitudes in the "naive non-Abelianization" approximation and powersuppressed corrections to $F_{M}\left(Q^{2}\right)$

Ahmedov, H. \& Dayi, Ö.F., Non-Abelian fractional supersymmetry in two dimensions

Akama, K. \& Hattori, T., Induced field theory on the brane world - gravity, extrinsic curvature, and gauge fields

Algin, A., Arik, M. \& Atakishiyev, N.M., SU(d)invariant multidimensional $q$ oscillators with bosonic degeneracy

Amelino-Camelia, G. \& Buccella, F., Testing $\kappa-$ Poincaré with neutral kaons

Añaños, G.N.J. \& Svaiter, N.F., The composite operator (CJT) formalism in the $\left(\lambda \varphi^{4}+\right.$ $\left.\eta \varphi^{6}\right)_{D=3}$ model at finite temperature

Anchishkin, D.V., Gavrilik, A.M. \& Iorgov, N.Z., $q$-boson approach to multiparticle correlations

Anchordoqui, L.A., Trobo, M.L., Vucetich, H. \& Zyserman, F., Gravitational memory of natural wormholes

\author{
A15(2000)2027 \\ A15(2000)465 \\ Anchordoqui, L.A., \\ Capozziello, S., Lambiase, G. \\ \& Torres, D.F., Radiation from \\ a uniformly accelerated charge \\ in the outskirts of a wormhole \\ throat
}

A15(2000) 15

A15(2000)1577

A15(2000)1419

A15(2000)1801

A15(2000)2017

A15(2000)1237

A15(2000)2119

A15(2000)2235

A15(2000)1637

A15(2000)429
Antunović, Ž., see Dželalija

Aquilano, R., Castagnino, M. \& Eiroa, E., Entropy gap and time asymmetry II

A15(2000)2219

A15(2000)465

A15(2000) 875

Arazi, A. \& Simeone, C., Wiggly strings in linearized Brans-Dicke gravity

Arik, M., see Algin

Atakishiyev, N.M., see Algin

Axenides, M., Floratos, E.G. \& Perivolaropoulos, L., Some dynamical effects of the cosmological constant

Azevedo, S., Self-force on timedependent pointlike source in the space of a disclination

Bagchi, B. \& Bhattacharyya, P., Determining the parameters of $\eta \eta^{\prime}$ mixing in the framework of anomalous Ward identities

Bagchi, B., Ganguly, A., Bhaumik, D. \& Mitra, A., Higher derivative supersymmetry, a modified CrumDarboux transformation and coherent state

A15(2000)309(E)

Bagchi, B. \& Bhaumik, D., A reappraisal of two-mode squeezing and intermode coupling

A15(2000)1369

A15(2000) 1237

A15(2000)1237

A15(2000)1541

A15(2000)1689

A15(2000)167

Balachandran, A.P.,

Govindarajan, T.R. \& Ydri, B., The fermion doubling problem and noncommutative geometry

A15(2000) 1279

Baleanu, D. \& Bașkal, S., Geometrization of the Lax pair tensors

Banerjee, N., see Sen

Bannur, V.M., see Sahu

Basu, D., Are some host galaxies of SNe Ia actually blueshifting?

A15(2000)825

Başkal, S., Dirac gamma matrices as representations of the algebra of little groups
$\mathbf{A 1 5}(2000) 1503$
$\mathbf{A 1 5}(2000) 1409$
$\mathbf{A 1 5}(2000) 775$

A15(2000)833
A15(2000)2357 
Başkal, S., see Baleanu

Begzhanov, R.B. \&

Sharafiddinov, R.S., On the $\mathrm{CP}$-symmetry in the neutrino scattering by nuclei

Belhaj, A. \& Saidi, E.H., On hyper-Kahler singularities

Beyer, M., see Bodoor

Bezerra, V.B., see Furtado

Bezerra, V.B., see de Assis

Bezerra, V.B., see Spinelly

Bhatia, V.B., see Jain

Bhatnagar, V., see Randhawa

Bhattacharyya, D.P., see Gupta

Bhattacharyya, P., see Bagchi

Bhattacharyya, R. \& Gangopadhyay, D., Duality in equations of motion from space-time dependent

Lagrangians

Bhaumik, D., see Bagchi

Bhaumik, D., see Bagchi

Bizdadea, C., Note on twodimensional nonlinear gauge theories

Bodoor, K., Weber, H.J., Frederico, T. \& Beyer, M., Violations of Lorentz covariance in light front quark models

Bose, S.K. \& Schulze-Halberg, A., Exact solution of the Dirac equation for a singular central power potential

Böttner, T., Ketov, S.V. \& Lau, T., Manifestly $N=3$ supersymmetric Euler-Heisenberg action in light-cone superspace

Bourrely, C., Soffer, J. \& Wu, T.T., $\gamma \gamma$ total cross-section at high energies

Boz, M., Demir, D.A. \& Pak, N.K., Q-ball formation in the MSSM with explicit CP violation

Boz, M. \& Kirca, Z., The light scalar bottom quark and $c \rightarrow$ $u \gamma$ decay

Brandt, F.T., McKeon, D.G.C. \& Sherry, T.N., Supersymmetry in $2+2$ dimensions

Bratchikov, A.V., Generalized Abelian coset conformal field theories
A15(2000)1503

A15(2000)557

A15(2000) 1767

A15(2000)2191

A15(2000) 253

A15(2000)945

A15(2000)1961

A15(2000)41

A15(2000) 2363

A15(2000) 1567

A15(2000) 167

A15(2000)901

A15(2000)309(E)

A15(2000) 825

A15(2000)2047

A15(2000)2191

A15(2000)1583

A15(2000)587

A15(2000) 9

A15(2000)517

A15(2000)2345

A15(2000)1349

A15(2000)809
Brenig, L., see Gunzig

A15(2000)1363

Brihaye, Y. \& Desoil, M., Gravitating (bi-)sphalerons

Brihaye, Y., Quasi-exactly solvable matrix Schrödinger operators

Brodimas, G., see Jannussis

Buccella, F., see Abud

Buccella, F., see AmelinoCamelia

Buchbinder, I.L., Cvetič, M. \& Petrov, A.Yu., Implication of decoupling effects for oneloop corrected effective actions from superstring theory

Burdík, Č., Pashnev, A. \& Tsulaia, M., Auxiliary representations of Lie algebras and the BRST constructions Bytsenko, A.A., Gonçalves, A.E. \& Williams, F.L., ChernSimons invariants of closed hyperbolic three-manifolds

Calixto, M., Promoting finite to infinite symmetries: the $(3+1)$ dimensional analogue of the Virasoro algebra and higherspin fields

Camacho, A., Group-theoretical structure of quantum measurements and equivalence principle

A15(2000) 889

A15(2000)1647

A15(2000) 1385

A15(2000) 15

A15(2000)2119

A15(2000)783

A15(2000)281

A15(2000)1031

A15(2000)939

Capozziello, S., De Martino, S., De Siena, S. \& Illuminati, F., Phenomenological scaling laws relating the observed galactic dimensions to the microscopic fundamental scales

Capozziello, S., see

Anchordoqui

Cariñena, J.F. \& Ramos, A., The partnership of potentials in quantum mechanics and shape invariance

Castagnino, M., see Aquilano

Cavaglià, M., A note on Weyl transformations in twodimensional dilaton gravity

Cea, P., Fogli, G.L. \& Tedesco, L., Influence of the magnetic field on the fermion scattering off bubble and kink walls
A15(2000)1063

A15(2000)2219

A15(2000) 1079

A15(2000)875

A15(2000)2113

A15(2000)1755 
Chang, Z. \& Guo, H.-Y., Symmetry realization, Poisson kernel and the AdS/CFT correspondence

Charles, F., see Dželalija

Chaves, M. \& Morales, H., Grand unification using a generalized Yang-Mills theory

Chen, J.-S. \& Li, J.-R., NonAbelian permittivity of quarkgluon plasma due to the selfinteraction of field

Chen, Y., He, B. \& Wu, J.-M., Non-Abelian Stokes theorem and computation of Wilson loop

Chen, Y., He, B., Lin, H. \& Wu, J.-M., SU(2) gluon propagator on a coarse anisotropic lattice Chiapparini, M., see de Oliveira Chiapparini, M., see Taurines

Chiou-Lahanas, C., Diamandis, G.A., Georgalas, B.C., Kapella-Ekonomou, A. \& Maintas, X.N., Static and nonstatic quantum effects in two-dimensional dilaton gravity

Cho, G.-C., Looking for $Z^{\prime}$ bosons in supersymmetric $E_{6}$ models through electroweak precision data

Choudhury, T.R., see

Padmanabhan

Clark, T.E. \& Love, S.T., Twisted boundary conditions and matching to the effective four-dimensional theory

Clark, T.E., Love, S.T. \& Nowling, S.R., Charged spin$1 / 2$ particle in an arbitrary magnetic field in two spatial dimensions: A supersymmetric quantum mechanical system

Cleaver, G.B., Faraggi, A.E., Nanopoulos, D.V. \& Walker, J.W., Non-Abelian flat directions in a minimal superstring standard model

Constantinescu, R. \& Ionescu, C., The $\mathrm{sp}(3)$ BRST symmetry for reducible dynamical systems
Cooperstock, F.I., The behavior of rotating bodies that emit gravitational waves in general relativity

Cotăescu, I.I. \& Visinescu, M., Schrödinger quantum modes on the Taub-Nut background A15(2000)197 Cristofano, G., Maiella, G. \& Marotta, V., A twisted conformal field theory description of the quantum Hall effect

Cristofano, G., Maiella, G. \& Marotta, V., A conformal field theory description of the paired and parafermionic states in the quantum Hall effect

A15(2000) 2245

A15(2000) 1529

A15(2000)1789

Cvetič, G. \& Yu, J.-Y., BorelPadé vs Borel-Weniger method: a QED and a QCD example

Cvetič, M., see Buchbinder

Czerhoniak, P., Force in kappa-deformed relativistic dynamics

A15(2000)1627

da Cruz, W. \& de Oliveira, R., Fractal index, central charge and fractons

Dadhich, N.K., see Joshi

Dafounansou, O., see Kachkachi

Dayi, Ö.F., see Ahmedov

de Assis, J.G., de Oliveira, S.B. \& Bezerra, V.B., Loop variables and global characterization of conical space-times

A15(2000)1137

de Berredo-Peixoto, G., PennaFirme, A. \& Shapiro, I.L., One-loop divergences of quantum gravity using conformal parametrization de Leo, S., Ducat, G. \& Rotelli, P., Remarks upon the mass oscillation formulas

De Martino, S., see Capozziello de Oliveira, J.C.T., Kyotoku, M., Chiapparini, M., Rodrigues, H. \& Duarte, S.B., Delta matter formation in dense asymmetric nuclear medium

de Oliveira, R., see da Cruz

A15(2000)1037
A15(2000)1529

A15(2000)1107

A15(2000)145

A15(2000) 547

A15(2000)1679

A15(2000)1227

A15(2000)783

$\mathbf{A 1 5}(2000) 1823$

A15(2000)1931

A15(2000)991

A15(2000)417

A15(2000)1801

A15(2000)945

A15(2000)2335

A15(2000)2057

A15(2000)1063

A15(2000)945
A15(2000)1931 
De Siena, S., see Capozziello

Deguchi, S. \& Mandal, B.P., A superspace formulation of Abelian antisymmetric tensor gauge theory

Demir, D.A., see Boz

Demkin, P., Simplicial complexes and the semiclassical limit of quantum gravity

Desai, B.R. \& Vaucher, A.R., Bootstrap of the scalar sector in top-antitop scattering amplitude

Desai, B.R. \& Vaucher, A.R., Higgs propagator in the presence of strong dynamics in the electroweak sector

Desoil, M., see Brihaye

Dev, S. \& Sharma, J.D., Resolution of the Be/B solar neutrino flux anomaly in the resonant spin-flavor precession scenario with twisting solar magnetic fields

Dev, S. \& Sharma, J.D., The status of the solar neutrino problem in the resonant spinflavor precession scenario with twisting solar magnetic fields

Dey, J., see Ray

Dey, J., see Ray

Dey, M., see Ray

Dey, M., see Ray

Diamandis, G.A., see ChiouLahanas

Diaz-Cruz, J.L., Hernandez, J.M. \& Toscano, J.J., Impact of the bounds on Higgs mass and $m_{W}$ on effective theories

Djeghloul, N. \& Tahiri, M., An alternative construction of the quantum action for supergravity

Dobson, Jr., P.N., see Mak

Doff, A. \& Pisano, F., Minimal extended flavor groups, matter fields chiral representations, and the flavor question

Dolan L. \& Nappi, C.R., The Ramond-Ramond self-dual five-form's partition function on $T^{10}$
A15(2000)1063

A15(2000)965

A15(2000) 517

A15(2000)841

A15(2000)1429

A15(2000)1483

A15(2000) 889

A15(2000)351

A15(2000) 1445

A15 (2000)683

A15(2000)1301

A15(2000)683

A15(2000)1301

A15(2000)1627

A15(2000)1377

A15(2000)1307

A15(2000) 2153

A15(2000)1471

A15(2000)1261
Dong, F.-X. \& Zhou, X.-J., $\gamma \gamma$ $\rightarrow \gamma Z$ Scattering and its related processes

Duarte, S.B., see de Oliveira

Ducat, G., see de Leo

Dvali, G., Gabadadze, G. \& Porrati, M., On sub-millimeter foces from extra dimensions

Dymnikova, I. \& Khlopov, M., Decay of cosmological constant as Bose condensate evaporation

Dželalija, M., Antunović, Ž., Abdullin, S. \& Charles, F., Low luminosity SUSY searches at large $\tan \beta$ in CMS

Éboli, O.J.P., Gonzalez-Garcia, M.C. \& Novaes, S.F., Indirect constraints on the triple gauge boson couplings from $Z \rightarrow b \bar{b}$ partial width: an update

Eiroa, E., see Aquilano

El Kinani, E.H., Hidden quantum group symmetry in 2-D fractional supersymmetry

El Rhalami, A., see Kachkachi

Eremin, S. \& Ivanov, E., Holomorphic effective action of $N=2$ SYM theory from harmonic superspace with central charges

Escobar, C.O., see Santos

Falcone, D., see Abud

Fan, H.-Y. \& Yu, G.-C., Radon transformation of the Wigner operator for two-mode correlated system in generalized entangled state representation

Fan, H.-Y., Weyl-ordered polynomials studied by virtue of the IWWOP technique

Fang, X.-Y., Guo, S.-H. \& Liu, J.-M., Coupled cluster expansions for $(2+1)$-dimensional $\mathrm{U}(1)$ lattice gauge theory with improved Hamiltonian

Faraggi, A.E., see Cleaver

Fateev, V.A., Normalization factors in conformal field theory and their applications Fedoruk, S. \& Zima, V.G., Massive superparticle with tensorial central charges
A15(2000) 2387

A15(2000) 1529

A15(2000)2057

A15(2000)1717

A15(2000)2305

A15(2000)465

A15(2000)1

A15(2000)875

A15(2000)2139

A15(2000)417

A15(2000)1859

A15(2000)1833

A15(2000) 15

A15(2000)499

A15(2000)2297

A15(2000)737

A15(2000)1191

A15(2000)259

A15(2000)2281 
Field, J.H. \& Sciarrino, D., A study of the LEP and SLD measurements of $A_{\mathrm{b}}$

Figueiredo, A., see Gunzig

Filho, E.D. \& Ricotta, R.M., Induced variational method from supersymmetric quantum mechanics and the screened Coulomb potential

Finkelstein, R.J., Quantum groups and field theory

Fiziev, P.P., A minimal model for dilatonic gravity

Floratos, E.G., see Axenides

Foerster, A., see Hibberd

Fogli, G.L., see Cea

Frederico, T., see Bodoor

Freund, M. \& Ohlsson, T., Matter enhanced neutrino oscillations with a realistic earth density profile

Fukuma, M. \& Sakai, T., Comment on ambiguities in the holographic Weyl anomaly

Furtado, C., Bezerra, V.B. \& Moraes, F., Aharonov-Bohm effect for bound states in Kaluza-Klein theory

Furuta, K. \& Inami, T., Ultraviolet property of noncommutative Wess-

Zumino-Witten model

Gabadadze, G. \& Kakushadze, Z., Zero-brane matrix mechanics, monopoles and membrane approach in QCD

Gabadadze, G., see Dvali

Gaeta, G., On the cosmological implications of the Calogero conjecture

Gangopadhyay, D., see

Bhattacharyya

Ganguly, A., see Bagchi

Ganguly, A., Associated Lamé equation, periodic potentials and $\operatorname{sl}(2, R)$

Garcia de Andrade, L.C., see Sivaram

Garcia de Andrade, L.C., Averaged weak energy condition violation for wormhole geometries with torsion
A15(2000)761

A15(2000)1363

A15(2000)1253

A15(2000)1709

A15(2000)1977

A15(2000) 1541

A15(2000) 133

A15(2000) 1755

A15(2000)2191

A15(2000)867

A15(2000)1703

A15(2000)253

A15(2000)997

A15(2000)293

A15(2000) 1717

A15(2000)1329

A15(2000)901

A15(2000)309(E)

A15(2000)1923

A15(2000)1275

A15(2000)1321
García, A., Huerta, R. \& Sánchez-Colón, G., A priori mixing of mesons and the $|\Delta I|$ $=1 / 2$ rule in $K \rightarrow \pi \pi$

A15(2000)1749

Gavrilik, A.M., see Anchishkin

Geng, C.Q., Lih, C.C. \& Zhang, W.-M., Study of radiative leptonic $D$ meson decays

Georgalas, B.C., see ChiouLahanas

Georgopoulos, G., Petridis, A. \& Vassiliou, M., Discrete wavelet analysis for multiparticle production experiments

Ghazal, A.Sh., see Ismail

Ghinculov, A. \& Yao, Y.-P., Momentum expansion of massive two-loop Feynman graphs around a finite value Ghinculov, A. \& Yao, Y.-P., Exact $0\left(g^{2} \alpha_{s}\right)$ top decay width from general massive twoloop integrals

Ghinculov, A. \& Yao, Y.-P., Exact nonfactorizable $\mathrm{O}\left(\alpha_{s} g^{2}\right)$ two-loop contribution to $Z \rightarrow$ $b \bar{b}$

A15(2000) 1637

A15(2000)2087

A15(2000)1627

A15(2000)1051

A15(2000)2315

A15(2000)509

A15(2000)925

A15(2000)1967

Ghosh, D.K., Poulose, P. \& Sridhar, K., Seeking TeV scale quantum gravity at an $e \gamma$ collider

A15(2000) 475

Gieres, F., Nieder, H., Pisar, T., Popp, L. \& Schweda, M., Interacting six-dimensional topological field theories

Gilkey, P. \& Park, J.H., Heat content asymptotics of an inhomogeneous time-dependent processes

Gill, P.S., see Randhawa

Glück, M. \& Reya, E., Phenomenology of the flavorasymmetry in the light-quark sea of the nucleon

Goldman, T., Stephenson, G.J., Jr. \& McKellar, B.H.J., Implications of quark-lepton symmetry for neutrino masses and oscillations

Gomshi Nobary, M.A. \& O'sati, T., Fragmentation functions for $B_{c}$ and $B_{c}^{*}$ mesons with definite polarization
A15(2000)1165

A15(2000)2363

$\mathbf{A 1 5}(2000) 439$

A15(2000)791

A15(2000)883

A15(2000)455 
Gonçalves, A.E., see Bytsenko

Gonzalez-Garcia, M.C., see Éboli

Gorbunov, D.S., On electroweak baryogenesis in gauge mediated models with messengermatter mixing

Govindarajan, T.R., see

Balachandran

Gunzig, E., Brenig, L., Figueiredo, A. \& Rocha Filho, T.M., A no-chaos theorem for non-minimally coupled scalar field cosmology and a new cosmogenesis scenario

Guo, H.-Y., see Chang

Guo, S.-H., see Fang

Gupta, M., see Randhawa

Gupta, N. \& Bhattacharyya, D.P., Investigation on the neutrino induced muons from active galactic nuclei

Gurappa, N., Panigrahi, P.K., Raju, T.S. \& Srinivasan, V., Quantum equivalent of the Bertrand's theorem

Haba, N. \& Suzuki, T., Is $U_{e 3}$ really related to the solar neutrino solutions?

Haba, Z., Decoherence by relic gravitons

Halyo, E., Mirage gauge coupling unification and $\mathrm{TeV}$ scale strings

Halyo, E., Supergravity on $A d S_{5 / 4} \times$ Hopf fibrations and conformal field theories

Harikumar, E. \& Sivakumar, M., On the equivalence between topologically and nontopologically massive Abelian gauge theories

Harko, T., see Mak

Hartmann, B., Kleihaus, B. \& Kunz, J., Dyons with axial symmetry

Hattori, T., see Akama

$\mathrm{He}$, B., see Chen

$\mathrm{He}$, B., see Chen

Hernandez, J.M., see Diaz-Cruz Hibberd, K., Roditi, I., Links, J. \& Foerster, A., Bethe ansatz solution of the closed anisotropic supersymmetric $U$ model with quantum supersymmetry
A15(2000)1031

$\mathbf{A 1 5}(2000) 1$

A15(2000)207

A15(2000)1279

A15(2000) 1363

A15(2000) 407

A15(2000) 737

A15(2000)2363

A15(2000) 1567

A15(2000)1851

A15(2000)2257

A15(2000)1519

A15(2000)343

A15(2000)397

A15(2000) 121

A15(2000)2153

A15(2000)1003

A15(2000)2017

A15(2000) 1127

A15(2000)2245

A15(2000)1377

$\mathbf{A 1 5}(2000) 133$
Hong, S.-T., Kim, Y.-W. \& Park, Y.-J., Symmetries of SU(2) Skyrmion in Hamiltonian and Lagrangian approaches

Hong, S.-T. \& Park, Y.-J., BFT Hamiltonian embedding for SU(3) Skyrmion

Hong, S.-T., Kim, W.T. \& Park, Y.-J., Improved Dirac quantization of a free particle

Hssaini, M., Kessabi, M., Maroufi, B. \& Sedra, M.B., On the harmonic superspace language adapted to the Gelfand-Dickey algebra of differential operators

Huang, M., see Zhuang

Huerta, R., see García

Ichinose, I. \& Nagao, K., GrossNeveu model with overlap fermions

Ikemori, H., Kitakado, S., Otsu, H. \& Sato, T., Berry connections and induced gauge fields in quantum mechanics on sphere

Illuminati, F., see Capozziello

Inami, T., see Furuta

Ionescu, C., see Constantinescu

Iorgov, N.Z., see Anchishkin

Ismail, M. \& Ghazal, A.Sh., Microscopic study of the effect of hexadecapole deformation of both fusion cross-section and barrier distribution

Ivanov, E., see Eremin

Jain, D., Panchapakesan, N., Mahajan, S. \& Bhatia, V.B., Constraints on galaxy evolution through gravitational lensing statistics

Jannussis, A. \& Brodimas, G., Connection between $Q$ algebras and noncanonical Heisenberg algebras

Jinzenji, M., Completion of the conjecture: quantum cohomology of Fano hypersurfaces

Jinzenji, M., Virtual GromovWitten invariants and the quantum cohomology rings of general type projective hypersurfaces
A15(2000)55

A15(2000)913

A15(2000)1915

A15(2000)651

A15(2000) 1617

A15(2000)1749

A15(2000)857

A15(2000)1203

A15(2000) 1063

A15(2000)997

A15(2000) 1037

A15(2000) 1637

A15(2000)2315

A15(2000)1859

A15(2000)41

A15(2000)1385

A15(2000)101

A15(2000)629 
Joglekar, S.D., Green's functions in axial- and Lorentztype gauges and BRS transformations

Joglekar, S.D. \& Misra, A., Wilson loop and the treatment of axial gauge poles

Joglekar, S.D. \& Misra, A., A derivation of the correct treatment of $\frac{1}{(\eta \cdot k)^{p}}$ singularities in axial gauges

Joshi, P.S., Dadhich, N.K. \& Maartens, R., Gamma-ray bursts as the birth-cries of black holes

Kachkachi, M., Dafounansou, O. \& El Rhalami, A., The chirally split diffeomorphism anomaly and the $\mu$-holomorphic projective connection Kakushadze, Z., see Gabadadze

Kakushadze, Z., "Self-tuning" and conformality

Kakushadze, Z. \& Langfelder, P., Gravitational Higgs mechanism

Kamani, D., Moving mixed branes in compact space-time

Kapella-Ekonomou, A., see Chiou-Lahanas

Kaushal, R.S., Possibility of a geometric constraint in the Schrödinger quantum mechanics

Kessabi, M., see Hssaini

Ketov, S.V., see Böttner

Khanal, U., Dynamics of strings with Gaussian density and tension

Khlopov, M., see Dymnikova

Kim, W.T. \& Oh, J.J., Noncommutative open strings from Dirac quantization

Kim, W.T., see Hong

Kim, Y.-W., see Hong

Kirca, Z., see Boz

Kitakado, S., see Ikemori

Kleihaus, B., see Hartmann

Kling, A., Kreuzer, M. \& Zhou, J.-G., SU(2) WZW D-branes and quantized world-volume U(1) flux on $S^{2}$

Kogan, I.I. \& Wheater, J.F., What does $E_{8}$ know about 11 dimensions?

Kogan, I.I. \& Tsvelik, A.M., Logarithmic operators in the

A15(2000)245

A15(2000)541, 1539(E)

A15(2000)1347(E)

A15(2000)991

A15(2000) 417

A15(2000)293

A15(2000)1879

A15(2000)2265

A15(2000) 1655

A15(2000)1627

A15(2000)1391

A15(2000)651

A15(2000)587

A15(2000)675

A15(2000)2305

A15(2000)1597

A15(2000)1915

A15(2000) 55

A15(2000)2345

A15(2000)1203

A15(2000) 1003

A15(2000)2069

A15(2000)851 theory of plateau transition

Kondo, K.-I. \& Taira, Y., NonAbelian Stokes theorem and quark confinement in SU(3) Yang-Mills gauge theory

Koyama, H., Nambu, Y. \& Tomimatsu, A., Vacuum polarization of massive scalar fields on the black hole horizon

Kreuzer, M., see Kling

Kunz, J., see Hartmann

Kuznetsov, A.V., Mikheev, N.V. \& Rumyantsev, D.A., Lepton pair production by highenergy neutrino in an external electromagnetic field

Kyotoku, M., see de Oliveira

Lambiase, G., see Anchordoqui

Langfelder, P., see Kakushadze

Lau, T., see Böttner

Leitgeb, R., Rant, J., Schweda, M. \& Zerrouki, H., Generalized 2-D BF model quantized in the axial gauge

Li, D.-M., Yu, H. \& Shen, Q.$X$., Study on hadronic decay of the lowest lying glueballs with $J^{\text {PC }}=$ even $^{++}$to two pseudoscalar mesons

Li, D.-M., Yu, H. \& Shen, Q.X., Modification of Kawai model about the mixing of the pseudoscalar mesons

A15(2000)931

A15(2000)367

A15(2000) 815

A15(2000)2069

A15(2000) 1003

A15(2000) 573

A15(2000)1529

A15(2000)2219

A15(2000)2265

A15(2000) 587

A15(2000)483

A15(2000) 723

Li, D.-M., Yu, H. \& Shen, Q.$X$., On the mixing of the scalar mesons $f_{0}(1370), f_{0}$ (1500) and $f_{0}(1710)$

Li, J., see Wong

Li, J.-R., see Chen

Li, W.B., see Yue

Li, W.-B., see Yue

Li, Xiang \& Zhao, Zheng, Entropy of a nonstatic black hole

Lih, C.C., see Geng

Lima, C.L., see Timóteo

Lin, H., see Chen

Lindebaum, R.J., see Tupper

Links, J., see Hibberd
A15(2000)1213

A15(2000)1781

A15(2000)2011

A15(2000)1843

A15(2000)361

A15(2000)2183

A15(2000)1739

A15(2000)2087

A15(2000) 219

A15 (2000) 2245

A15(2000)1221

A15(2000)133 
Lipatov, A.V. \& Zotov, N.P., Study of BFKL gluon dynamics in heavy quarkonium photoproduction at HERA

Lipatov, A.V., Saleev, V.A. \& Zotov, N.P., Heavy quark photoproduction in the semihard QCD approach and the unintegrated gluon distribution

Liu, C., Electroweak symmetry breaking due to confinement

Liu, F.-H., Formulation of negatively charged particle rapidity distribution in nucleus-nucleus collisions at high energy

Liu, J.-M., see Fang

Loewe, M. \& Valenzuela, C., Thermal renormalons in scalar field theory

Lokhanin, M.V., see Svetovoy

Lokhanin, M.V., see Svetovoy

Long, H.N., see Soa

Love, S.T., see Clark

Love, S.T., see Clark

Lu, G.-R., see Yue

Ma, J.P., A study of gluon propagator on coarse lattice

Maartens, R., see Joshi

Machet, B., Extending the standard model: an upper bound for a neutrino mass from the rare decay $K^{+} \rightarrow$ $\pi^{+} v \bar{v}$

Mahajan, S., see Jain

Maharaj, S.D., see Sharma

Maiella, G., see Cristofano

Maiella, G., see Cristofano

Maintas, X.N., see ChiouLahanas

Mak, M.K., Dobson, Jr., P.N. \& Harko, T., Maximum massradius ratio for compact general relativistic objects in Schwarzschild-de Sitter geometry

Malheiro, M., see Taurines

Malik, R.P., New local symmetry for QED in two dimensions

Mamedova, Y.V., see Agaev

Mandal, B.P., see Deguchi
$\mathbf{A 1 5}(2000) 695$

A15(2000)1727

A15(2000) 525

A15(2000)1497

A15(2000)737

A15(2000)1181

A15(2000)1013

A15(2000) 1437

A15(2000) 23

A15(2000)1137

A15(2000)2105

A15(2000)2183

A15(2000)229

A15(2000)991

A15(2000) 579

A15(2000) 41

A15(2000)1341

A15(2000) 547

A15(2000) 1679

A15(2000)1627

A15(2000)2153

A15(2000) 1789

A15(2000) 2079

A15(2000)1419

A15(2000)965
Manvelyan, R. \& Mkrtchyan, R., Towards $\mathrm{SO}(2,10)-$ invariant M-theory: multiLagrangian fields

A15(2000)747

Marino, E.C. \& Sasaki, D.G.G., Quantum magnetic vortices at finite temperature in $(3+1)$-D

Marnelius, R., On the quantum BRST structure of classical mechanics

Marotta, V., see Cristofano

Marotta, V., see Cristofano

Maroufi, B., see Hssaini

Masuda, T., Normalized Weyltype $\star$-product on Kähler manifolds

Matsuki, T. \& Shiotani, M., Effective couplings of dynamical Nambu-Goldstone bosons with elementary fermions

Matsuyama, T. \& Nagahiro, H., Dynamical mass and parity condensate in varying topological mass

Mayer, D., Ushveridze, A. \& Walczak, Z., On time-dependent quasi-exactly solvable problems

McKellar, B.H.J., see Goldman McKeon, D.G.C. \& Ord, G.N., Time reversal in stochastic processes and the Dirac equation II: coupling to an external electromagnetic field McKeon, D.G.C., see Brandt

Menaa, M. \& Tahiri, M., A note on topological 4-D gravity and its metric approach

Miguchi, H., see Abe

Mikheev, N.V., see Kuznetsov

Mikheev, N.V. \& Narynskaya, E.N., Neutrino-electron processes in a dense magnetized plasma

Misra, A., see Joglekar

Misra, A., see Joglekar

Mitra, A., see Bagchi

Mkrtchyan, R., see Manvelyan

Mohanta, R., Study of direct CP asymmetry in $B^{+} \rightarrow K^{0} \pi^{+}$

Mora, C., see Pimentel

Moraes, F., see Furtado

Morales, H., see Chaves

\section{A15(2000)731}

A15(2000)1665

A15(2000) 547

A15(2000) 1679

A15(2000)651

A15(2000)2177

A15(2000)709

A15(2000)2373

A15(2000) 1243

A15(2000)439

A15(2000)595

A15(2000)1349

A15(2000)565

A15(2000) 445

A15(2000)573

A15(2000)1551

A15(2000)541,

1539(E)

A15(2000)1347(E)

A15(2000)309(E)

A15(2000)747

A15(2000)599

A15(2000)333

A15(2000)197
A15(2000) 253 
Mori, F. \& Natale, A.A., Constraining models with superlight gravitino, scalar and pseudoscalar particles

Mostafazadeh, A. \& Samani, K.A., Topological symmetries

Mostafazadeh, A., Comment on the possibility of a geometric constraint in the Schrödinger quantum mechanics

Mukherjee, S., see Sharma

Mukhtarov, A.I., see Agaev

Muratori, B., see Adam

Muta, T., see Abe

Nagahiro, H., see Matsuyama

Nagao, K., see Ichinose

Nambu, Y., see Koyama

Nanopoulos, D.V., see Cleaver

Nappi, C.R., see Dolan

Narynskaya, E.N., see Mikheev

Nash, C., see Adam

Natale, A.A., see Mori

Nesterenko, A.V., Investigation of a new analytic running coupling in QCD

Nieder, H., see Gieres

Nieto, J.A. \& Yee, C.M., pbrane action from gravidilaton effective action

Nitta, M., A note on supersymmetric WZW term in four dimensions

Nittoh, K.-I., A note on the quadratic divergence in hybrid regularization

Nojiri, S. \& Odintsov, S.D., Weyl anomaly from Weyl gravity

Novaes, S.F., see Éboli

Nowling, S.R., see Clark

O'sati, T., see Gomshi Nobary

Odintsov, S.D., see Nojiri

Oh, J.J., see Kim

Ohlsson, T., see Freund

Okun, L.B. \& Tsukerman, I.S., Comment on equal velocity assumption for neutrino oscillations

Okun, L.B., Photons and static gravity

Okun, L.B., A thought experiment with clocks in static gravity

Ord, G.N., see McKeon

Otsu, H., see Ikemori

\section{A15(2000)1099}

A15(2000) 175

A15(2000)2129

A15(2000)1341

A15(2000) 1419

A15(2000)1577

A15(2000) 445

A15(2000) 2373

A15(2000) 857

A15(2000) 815

A15(2000) 1191

A15(2000)1261

A15(2000)1551

A15(2000)1577

A15(2000)1099

A15(2000)2401

A15(2000)791

A15(2000)1611

A15(2000) 2327

A15(2000)955

A15(2000)1043

A15(2000) 1

A15(2000)2105

A15(2000) 455

A15(2000)1043

A15(2000)1597

A15(2000) 867

A15(2000)1481

A15(2000)1941

A15(2000)2007

A15(2000) 595

A15(2000)1203
Padmanabhan, T. \& Choudhury, T.R., The issue of choosing nothing: what determines the low energy vacuum state of nature?

$\operatorname{A15}(2000) 1813$

Pak, N.K., see Boz

Panchapakesan, N., see Jain

Paniak, L.D., Chern-Simons gravity, Wilson lines and large- $N$ dual gauge theories

Panigrahi, P.K., see Gurappa

Papantonopoulos, E. \& Pappa, I., Type 0 brane inflation from mirage cosmology

Pappa, I., see Papantonopoulos

Parikh, M.K., Phases of thermal super-Yang-Mills

Park, J.H., see Gilkey

Park, Y.-J., see Hong

Park, Y.-J., see Hong

Park, Y.-J., see Hong

Parthasarathy, R., Dual

Meissner effect in the infrared region of QCD with quarks

Pashnev, A., see Burdík

Pashnev, A.I., see Tkach

Pasupathy, J., A calculation of Higgs mass in the standard model

Penna-Firme, A., see de Berredo-Peixoto

A15(2000) 517

A15(2000) 41

A15(2000) 1117

A15(2000)1851

A15(2000)2145

A15(2000)2145

A15(2000)665

A15(2000)1165

A15(2000) 55

A15(2000)913

A15(2000)1915

A15(2000)2037

A15(2000)281

A15(2000) 1557

A15(2000)1605

A15(2000)2335

Perez Bergliaffa, S.E., Inhomogeneous multidimensional cosmologies

Perivolaropoulos, L., see Axenides

Peschanski, R., Hard jet probes in terms of colorless QCD dipoles

Pestov, A.B., The Weyl gauge theory and absolute parallelism

Pestov, A.B. \& Saha, B., On the natural gauge fields of manifolds

Petridis, A., see Georgopoulos

Petrov, A.Yu., see Buchbinder

Pierpaoli, E., Scott, D. \& White, M., Still flat after all these years!

Pimentel, L.O. \& Mora, C., Quantum cosmology and wormholes in some scalartensor theories
A15(2000)531

A15(2000)1541

A15(2000)1891

A15(2000)1697

A15(2000)1991

A15(2000)1051

A15(2000)783

A15(2000)1357

A15(2000)333 
Pinto, F., On the role of the Planck mass in cold atom beam scattering by a massive sphere

Pisano, F., see Doff

Pisar, T., see Gieres

Pisar, T., Rant, J. \& Zerrouki, H., A generalized $p$-form model in $D=3$

Pollock, M.D., Why is the hidden sector invisible?

Popp, L., see Gieres

Porrati, M., see Dvali

Poulose, P., see Ghosh

Radinschi, I., The energy distribution in a static spherically symmetric nonsingular black hole space-time

Radinschi, I., Energy of a conformal scalar dyon black hole

Raju, T.S., see Gurappa

Ramos, A., see Cariñena

Randhawa, M., Bhatnagar, V., Gill, P.S. \& Gupta, M., Implications of the unitarity triangle " $u c$ " for $J, \delta$ and $\left|\mathrm{V}_{\mathrm{CKM}}\right|$ elements

Rant, J., see Leitgeb

Rant, J., see Pisar

Rashdan, M., A Skyrme parametrization based on nuclear matter BHF calculations

Rath, B., New nonclassical solution on generalized Paul trap type: nonclassical study

Ray, K., Dey, J. \& Dey, M., Magnetic moment $\mu$ of $\Omega^{-}$baryon using Richardson potential in a relativistic Hartree-Fock (RHF) method

Ray, S., Dey, J. \& Dey, M., Density dependent strong coupling constant of QCD derived from compact star data

Raychaudhuri, A.K., A new singularity theorem in relativistic cosmology

Razmi, H. \& Abbassi, A.H., The fundamental commutator for massless particles

Ren Zhao, Zhang, L.C. \& Wu, Y.Q., The Nernst theorem and the entropy of a cylindrical black hole
$\mathbf{A 1 5}(2000) 2229$
$\mathbf{A 1 5}(2000) 1471$
$\mathbf{A 1 5}(2000) 791$

A15(2000)1147

A15(2000)2131

A15(2000)791

A15(2000)1717

A15(2000) 475

A15(2000)803

A15(2000) 2171

A15(2000) 1851

A15(2000)1079

A15(2000) 2363

A15(2000) 483

A15(2000)1147

A15(2000)1287

A15(2000)1071

A15(2000)683

$\mathbf{A 1 5}(2000) 1301$

A15(2000)391

A15(2000)2027

A15(2000)2165
Reya, E., see Glück

Rezaeian, A.H., see Setare

Ricciardi, G., see Abud

Ricotta, R.M., see Filho

Rocha Filho, T.M., see Gunzig

Roditi, I., see Hibberd

Rodrigues, H., see de Oliveira

Rosales, J.J., see Tkach

Rosu, H.C., Darboux class of cosmological fluids with timedependent adiabatic indices

Rotelli, P., see de Leo

Rumyantsev, D.A., see

Kuznetsov

Saha, B., see Pestov

Sahu, S. \& Bannur, V.M., Neutrino propagation in AGN environment

Saidi, E.H., see Belhaj

Sakai, T., see Fukuma

Saleev, V.A., see Lipatov

Samani, K.A., see

Mostafazadeh

Sánchez-Colón, G., see García

Sanders, D.A., Review of recent searches for rare and forbidden dilepton decays of charmed mesons

Santos, L.F. \& Escobar, C.O., Stochastic differential equations for the continuous spontaneous localization model

Sasaki, D.G.G., see Marino

Sato, T., see Ikemori

Schulze-Halberg, A., see Bose

Schweda, M., see Leitgeb

Schweda, M., see Gieres

Sciarrino, D., see Field

Scott, D., see Pierpaoli

Sedra, M.B., see Hssaini

Sen, A.A. \& Banerjee, N., Nonstatic global string in Brans-Dicke theory

Senovilla, J.M.M., $\left(\right.$ Super) ${ }^{n}$ energy for arbitrary fields and its interchange: conserved quantities

Sergeenko, M.N., Quasiclassical analysis of three-dimensional Schrödinger's equation and its solution

Setare, M.R. \& Rezaeian, A.H., Trace anomaly and Casimir effect
A15(2000)883

A15(2000)2159

A15(2000) 15

A15(2000) 1253

A15(2000) 1363

A15(2000) 133

A15(2000) 1529

A15(2000)1557

A15(2000)979

A15(2000)2057

A15(2000)573

A15(2000)1991

A15(2000)775

A15(2000)1767

A15(2000)1703

A15(2000)1727

A15(2000) 175

A15(2000)1749

A15(2000)1399

A15(2000)1833

A15(2000)731

A15(2000) 1203

A15(2000)1583

A15(2000) 483

A15(2000)791

A15(2000)761

A15(2000)1357

A15(2000)651

A15(2000)1409

A15(2000)159

A15(2000)83

A15(2000)2159 
Shapiro, I.L., see de BerredoPeixoto

Sharafiddinov, R.S., see Begzhanov

Sharma, J.D., see Dev

Sharma, J.D., see Dev

Sharma, R., Mukherjee, S. \& Maharaj, S.D., Scaling property in cold compact stars

Shen, Q.-X., see Li

Shen, Q.-X., see Li

Shen, Q.-X., see $\mathrm{Li}$

Shen, Y.-G., The fermionic entropy of spherically symmetric black holes

Sherry, T.N., see Brandt

Shiotani, M., see Matsuki

Simeone, C., see Arazi

Sivakumar, M., see Harikumar

Sivaram, C. \& Garcia de Andrade, L.C., Torsion modified plasma screening in astrophysics

Slad, L.M., Double symmetries in field theories

Soa, D.V. \& Long, H.N., Photoproduction of dilatons in an external magnetic field

Soffer, J., see Bourrely

Solovyov, A., On noncommutative solitons

Spinelly, J. \& Bezerra, V.B., Electrostatic in ReissnerNordström space-time with a conical defect

Sridhar, K., see Ghosh

Srinivasan, V., see Gurappa

Srivastava, Y.N. \& Widom, A., Obtaining the spin of the proton from the spins of the quarks

Stephenson, G.J., Jr., see Goldman

Sun, J.-F., see Yue

Suyama, T., Monopoles and black hole entropy

Suzuki, T., see Haba

Svaiter, N.F., see Añaños

Svetovoy, V.B. \& Lokhanin, M.V., Do the precise measurements of the Casimir force agree with the expectations?

Svetovoy, V.B. \& Lokhanin, M.V., Precise calculation of
A15(2000)2335

A15(2000)557

A15(2000)351

A15(2000) 1445

A15(2000) 1341

A15(2000)723

A15(2000) 1213

A15(2000) 1781

A15(2000) 1901

A15(2000)1349

A15(2000)709

A15(2000)1369

A15(2000) 121

A15(2000)1275

A15(2000)379

A15(2000) 23

A15(2000)9

A15(2000)2205

A15(2000)1961

A15(2000) 475

A15(2000)1851

A15(2000)2045

A15(2000)439

A15(2000) 2183

A15(2000) 271

A15(2000)2257

A15(2000)2235

A15(2000)1013 the Casimir force between gold surfaces

Tahiri, M., see Menaa

Tahiri, M., see Djeghloul

Taira, Y., see Kondo

Taurines, A.R., Vasconcellos, C.A.Z., Malheiro, M. \& Chiapparini, M., Neutron stars with parametrized meson couplings

Tedesco, L., see Cea

Timóteo, V.S. \& Lima, C.L., On the $q$-deformation of the NJL model

Tkach, V.I., Pashnev, A.I. \& Rosales, J.J., On the Schrödinger equation with time and its square root representation in the supersymmetric quantum mechanics

Tomimatsu, A., see Koyama

Torres, D.F., see Anchordoqui

Toscano, J.J., see Diaz-Cruz

Tramontano, F., see Abud

Trobo, M.L., see Anchordoqui

Tsukerman, I.S., see Okun

Tsulaia, M., see Burdík

Tsvelik, A.M., see Kogan

Tupper, G.B., Lindebaum, R.J. \& Viollier, R.D., Gauging the shadow sector with $\mathrm{SO}(3)$ Ushveridze, A., see Mayer Valenzuela, C., see Loewe Vasconcellos, C.A.Z., see Taurines

Vassiliou, M., see Georgopoulos

Vaucher, A.R., see Desai

Vaucher, A.R., see Desai

Viollier, R.D., see Tupper

Visinescu, M., see Cotăescu

Vucetich, H., see Anchordoqui

Walczak, Z., see Mayer

Walker, J.W., see Cleaver

Weber, H.J., see Bodoor

Wheater, J.F., see Kogan

White, M., see Pierpaoli

Widom, A., see Widom

Williams, F.L., see Bytsenko

Wisnivesky, D., Topological quantization of the magnetic flux

Wong, H.T. \& Li, J., Scintillation crystal detector for low energy neutrino physics
A15(2000)1437

A15(2000)565

A15(2000)1307

A15(2000)367

A15(2000) 1789

A15(2000) 1755

A15(2000)219

A15(2000) 1557

A15(2000)815

A15(2000)2219

A15(2000)1377

A15(2000) 15

A15(2000) 429

A15(2000)1481

A15(2000)281

A15(2000)931

A15(2000)1221

A15(2000) 1243

A15(2000)1181

A15(2000)1789

A15(2000)1051

A15(2000)1429

A15(2000)1483

A15(2000)1221

A15(2000) 145

A15(2000)429

A15(2000) 1243

A15(2000)1191

A15(2000)2191

A15(2000)851

A15(2000) 1357

A15(2000)2045

A15(2000)1031

A15(2000)1491

A15(2000)2011 
A15(2000) 1127

Wu, J.-M., see Chen

Wu, J.-M., see Chen

Wu, T.T., see Bourrely

Wu, Y.L., see Zhou

Wu, Y.Q., see Ren Zhao

Wu, Z.C., Quantum creation of a topological black hole

Xue, S.-S., Fine-tuning the lowenergy physics

Xulu, S.S., Møller energy for the Kerr-Newman metric

Yang, Z., see Zhen

Yang, Z., see Zhuang

Yao, Y.-P., see Ghinculov

Yao, Y.-P., see Ghinculov

Yao, Y.-P., see Ghinculov

Ydri, B., see Balachandran

Yee, C.M., see Nieto

Yoshioka, K., On fermion mass hierarchy with extra dimensions

Youm, D., A note on $(\mathrm{D}(p-2)$,

$\mathrm{D} p)$ bound state and noncommutative Yang-Mills theory

Yu, G.-C., see Fan

Yu, H., see Li

Yu, H., see Li

Yu, H., see Li

Yu, J.-Y., see Cvetič

Yue, C.X. \& Li, W.B., TC2 dynamics and single top quark production at high energy $e^{+} e^{-}$ colliders
A15(2000)2245

A15(2000)9

A15(2000) 185

A15(2000)2165

A15(2000)1589

A15(2000)1089

A15(2000)1511

A15(2000)617

A15(2000) 1617

A15(2000)509

A15(2000)925

A15(2000) 1967

A15(2000) 1279

A15(2000)1611

A15(2000)29

A15(2000)1949

A15(2000)499

A15(2000) 723

A15(2000) 1213

A15(2000) 1781

A15(2000) 1227

A15(2000)361
Yue, C.-X., Lu, G.-R., Sun, J.-F. \& Li, W.-B., Charged top-pion corrections to the top quark production at $\mathrm{LC}$ in TC2 theory

Zerrouki, H., see Leitgeb

Zerrouki, H., see Pisar

Zhang, L.C., see Ren Zhao

Zhang, W.-M., see Geng

Zhao, L., see Zhen

Zhao, W., see Zhuang

Zhao, Zheng see $\mathrm{Li}$

Zhen, Y., Zhao, L. \& Yang, Z., Integrating super-Liouville system without integration

Zhou, J.-G., see Kling

Zhou, X.-J., see Dong

Zhou, Y.F. \& Wu, Y.L., Separating different models from measuring $\alpha, \beta, \gamma$

Zhu, Z.-H., Gravitational lensing statistics as a probe of dark energy

Zhuang, P., Yang, Z., Huang, M. \& Zhao, W., An analytic solution of hydrodynamic equations with source terms in heavy-ion collisions

Zima, V.G., see Fedoruk

Zloshchastiev, K.G., Classical and quantum comparison of kink and bell solitons as zerobranes

Zotov, N.P., see Lipatov

Zotov, N.P., see Lipatov

Zyserman, F., see Anchordoqui
A15(2000)67

A15(2000)695

A15(2000) 1727

A15(2000)2183

A15(2000)483

A15(2000)1147

A15(2000)2165

A15(2000)2087

A15(2000)617

A15(2000) 1617

A15(2000)1739

A15(2000)617

A15(2000)2069

A15(2000)2387

A15(2000)185

A15(2000)1023

A15(2000) 1617

A15(2000)2281

A15(2000)429 